\title{
Hydrogen and helium production in structural materials by neutrons
}

\author{
R.C. Haight ${ }^{\mathrm{a}}$ \\ Los Alamos Neutron Science Center, Los Alamos, NM 87545, USA
}

\begin{abstract}
Hydrogen and helium are produced when energetic neutrons interact with materials, and these gases can lead to significant changes in materials properties such as embrittlement and swelling. Such effects have been seen in fission reactors and a significant effort has been made for the development of fusion reactors where the effects are expected to be larger because of the higher neutron energy. For the Advanced Fuel Cycle Initiative, new structural materials are proposed, and the amount of gas production must be known to assess the properties of these materials under radiation damage. We are measuring the production cross sections for these gases by neutrons in the energy range from threshold to $100 \mathrm{MeV}$ at the Los Alamos Neutron Science Center/Weapons Neutron Research (LANSCE/WNR) spallation source of fast neutrons. We measure the light charged particles (protons, deuterons, tritons, ${ }^{3} \mathrm{He}$ and alpha particles) emitted as a function of incident energy and angle and then integrate the angular distributions to obtain production cross sections. Results for the higher neutron energies are relevant to accelerated radiation damage facilities based on spallation neutron sources. The data measured for tantalum show a monotonic increase in the production cross sections of hydrogen and helium with neutron energy, whereas for iron and chromium, the cross sections flatten out above $50 \mathrm{MeV}$. Nuclear data evaluations often do not account well for the excitation functions.
\end{abstract}

\section{Introduction}

Neutron-induced radiation damage is well known in fission reactors and can lead to embrittlement and swelling of materials and possibly to other effects. Advanced reactors are expected to be even more susceptible to these effects due to the higher neutron fluences and to the higher neutron energy characteristic of fast reactors. Even higher neutron energies are expected in accelerator-based radiation damage facilities such as the Materials Test Station (MTS) proposed for the Los Alamos Neutron Science Center (LANSCE) where the spectrum will have $\sim 10 \%$ of the neutron flux above $10 \mathrm{MeV}$ and extending to over $100 \mathrm{MeV}$.

Principal causes of neutron-induced radiation damage are displacements of atoms in the material lattice and transmutation of the material to other elements. For the latter, the production of hydrogen and helium are generally thought to be particularly important. Introduction of these light gases can change the chemical properties of a material in the case of hydrogen and the physical stresses on the lattice because of build-up of helium inclusions. For a detailed understanding of radiation damage, the source terms must be quantified, that is, the number of displacements per atom (dpa) and the production of hydrogen and helium.

For accelerated radiation damage tests with acceleratorbased neutron sources, the observed damage needs to be related to that produced in other neutron fields with different neutron energy spectra. If the hydrogen and helium production relative to the dpa are the same, then one can have confidence that the accelerated test results of the changes in physical properties are relevant to the damage that will be produced at the different neutron energy spectra.

In this report, we describe the technique for measuring hydrogen and helium production cross sections as a function

\footnotetext{
a Presenting author, e-mail: haight@lanl.gov
}

of neutron energy from threshold to $100 \mathrm{MeV}$ using a continuous-in-energy spallation neutron source. The results are compared with data in evaluated data libraries where possible. These measurements were made in support of the Advanced Fuel Cycle Initiative of the US Department of Energy, Office of Nuclear Energy.

\section{Experiment}

To measure hydrogen and helium production cross sections, we detect light charged particles emitted in the reaction and measure their energy and angular distributions. These are double-differential data for proton, deuteron, triton, ${ }^{3} \mathrm{He}$ and alpha-particle production. Integration over both energy and angle gives the total production cross sections for each of these light isotopes. Adding all of the constituent isotopic data then gives the total hydrogen- and helium-production cross sections.

\subsection{Neutron source}

The WNR neutron source at a 30-degree production angle provided the continuous-in-energy source of neutrons. As was described in ref. [1], the $800-\mathrm{MeV}$ proton beam was incident on a water-cooled tungsten target $3 \mathrm{~cm}$ in diameter and $7 \mathrm{~cm}$ long. Typical currents of 1.6 microamperes were used with a micropulse spacing of 1.8 microseconds, a macropulse width of 625 microseconds and a rate of 40 macropulses per second. The micropulse width is approximately $200 \mathrm{ps,}$ much shorter than the overall time resolution of the experiment. The produced neutron spectrum is approximately that given in the WNR web site, wnr.lanl.gov/data. To reduce the number of neutrons at energies below the threshold for 
most of these reactions and to reduce the "gamma-flash" of prompt gamma rays from the target, absorbers of polyethylene $\left(\mathrm{CH}_{2}\right)(1.27 \mathrm{~cm})$ and lead $(1.27 \mathrm{~cm})$ were placed in the beam. Collimators defined the beam to be approximately $2.5 \mathrm{~cm}$ in diameter at the sample position. The collimators were designed with a double taper to reduce the number of scattered neutrons reaching the sample or the charged-particle detectors.

\subsection{Reaction chamber and detectors}

Samples were placed in a reaction chamber, $55.9 \mathrm{~cm}$ in inside diameter, centered 15.07 meters from the neutron source. Four samples arranged on a target ladder could be placed individually in the beam at the center of the chamber. One position was dedicated to a mixed ${ }^{228} \mathrm{Th}+{ }^{229} \mathrm{Th}$ source, which produced alpha particles from 4.5 to $8.3 \mathrm{MeV}$ for calibration purposes. Another position was dedicated to open or blank runs for background determination. An earlier version of the apparatus is described in ref. [2].

The detectors were charged-particle detector telescopes each consisting of a gas proportional counter, a 500-micron silicon surface-barrier detector, and a $3-\mathrm{cm}$ thick $\mathrm{CsI}(\mathrm{Tl})$ scintillator read out by a photodiode. For nearly all of the runs, $\mathrm{Ar}-\mathrm{CH}_{4}$ ("P10") or pure Xenon gas at pressures of approximately 20 torr filled the chamber including the gas counters. This procedure eliminated the possibility of leaks of counter gas into an evacuated chamber and allowed the experiments to be carried out with the chamber valved off from any gas supply. The gas quality deteriorated only slowly so that refreshing the gas was necessary only every two weeks or so. For these experiments, four detector telescopes were used simultaneously. They were usually placed at angles of 20,60, 90 and 135 degrees, but they could be placed at other angles to investigate better the angular distribution of the emitted charged particles.

The electronics were conventional. Timing signals were taken from the silicon surface barrier detectors. The "gammaflash" peak in the time spectra had a typical resolution of $3 \mathrm{~ns}$ FWHM. The energy of the neutron inducing the reaction was determined from the timing signals corrected for the time of flight of the charged particles from the sample to the detector. The energy of the detected charged particle was determined from the pulse height in the silicon detector, if the particle stopped there, or from a combination of silicon and CsI(Tl) signals. Calibration of the energy scale for the silicon detectors was carried out with a ${ }^{228} \mathrm{Th}+{ }^{229} \mathrm{Th}$ source. That for the $\mathrm{CsI}(\mathrm{Tl})$ detectors was determined from $\mathrm{n}$ - $\mathrm{p}$ scattering from a $\mathrm{CH}_{2}$ foil. The signal from the gas proportional counters was used to identify the particle type for alpha particles and low energy protons and deuterons. For these experiments, the resolution of the gas counters was not sufficient to separate isotopes of hydrogen ions or to separate ${ }^{3} \mathrm{He}$ from alpha particles. For the more penetrating protons and deuterons of higher energy, the silicon detector signal relative to the CsI(Tl) signal was used for particle identification, and here it was possible to separate protons from deuterons and these from tritons, and to separate the helium isotopes.

\subsection{Samples}

The materials tested were in the form of thin, metallic, elemental foils supplied by the Goodfellow Corp. [3]. A set of four thicknesses was used for each element in order to increase the efficiency of the experiment: thin foils were necessary for the measurement of low energy alpha particles, down to about $5 \mathrm{MeV}$. For the highly penetrating high energy protons, a much thicker foil could be used. Where possible, the foils were $10 \mathrm{~cm}$ in diameter to insure that the ring on which the foils were glued was well outside the beam and any beam halo. The foils were typically oriented at 55 degrees with respect to the beam direction in order to minimize the charged-particle energy loss and, at the same time, to increase their effective thickness to the neutron beam.

\subsection{Fluence normalization}

The neutron fluence on the sample was determined by a fission chamber [4] located 16.32 meters from the source, that is, downstream of the reaction chamber where the chargedparticle production was measured. For these measurements, we used the ${ }^{235} \mathrm{U}(\mathrm{n}, \mathrm{f})$ reaction and its cross section as given in ENDF/B-VI. A typical fluence spectrum is given in figure 1 where it is compared with an MCNPX calculation from first principles with the $800-\mathrm{MeV}$ proton beam incident on the tungsten target and including the $\mathrm{Pb}$ and $\mathrm{CH}_{2}$ attenuators [5]. Good agreement is found between measurement and calculation.

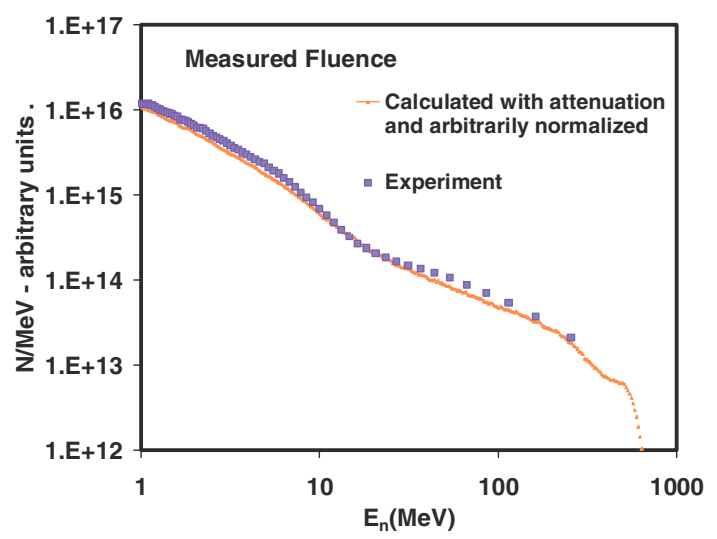

Fig. 1. Neutron fluence for one of the experimental runs determined with the ${ }^{235} \mathrm{U}$ fission chamber. The measurement is compared with an MCNPX calculation carried out from first principles [5].

\subsection{Data analysis}

Partial double-differential cross sections for emission of light charged particles for the different thickness samples were combined into full double differential cross sections, which were then integrated over angle and charged-particle energy and summed over particle type to give the total hydrogen and helium production cross sections. The double differential data will be reported elsewhere. 


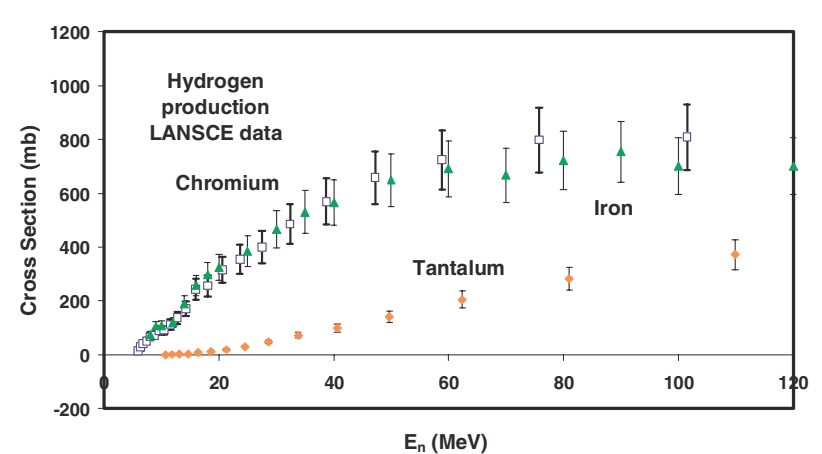

Fig. 2. Hydrogen production by neutrons on iron, chromium, and tantalum for neutron energies up to $100 \mathrm{MeV}$.

\section{Results and discussion}

Hydrogen and helium production cross sections for neutrons on iron, chromium and tantalum are shown in figures 2 and 3 for the full range of incident neutron energies, up to $100 \mathrm{MeV}$. The general trends are clear and show the differences between medium-mass nuclides $(\mathrm{Cr}, \mathrm{Fe})$ and the heavier-mass nuclide, Ta. The production cross sections for both hydrogen and helium rise quickly for $\mathrm{A} \sim 50-60$ nuclides and then flatten out around $40 \mathrm{MeV}$ above which energy the production cross sections are basically flat. For the heavier-mass nuclide ( $\mathrm{A}=$ 181 for Ta), these production cross sections are significantly less than for $A \sim 50-60$, but they continue to rise from threshold throughout this energy region.

We compare the measured results for helium production in iron with some of the literature values and with evaluated data libraries in figures 4 and 5. Our data are in good agreement with the monoenergetic value from Uppsala at $95 \mathrm{MeV}$ [6]. The experiment from Louvain [7] reported alpha particle production only for part of the spectrum, namely the high energy tail above the evaporation peak. At the lower energies, our data agree with helium accumulation data at $9.85 \mathrm{MeV}$ [8] and with charged-particle production data near $14 \mathrm{MeV}$, e.g., [9]. Because the residual nuclei for helium production, namely ${ }^{53} \mathrm{Cr}$ from ${ }^{56} \mathrm{Fe}(\mathrm{n}, \alpha)$ and ${ }^{52} \mathrm{Cr}$ from ${ }^{56} \mathrm{Fe}(\mathrm{n}, \mathrm{n}$ 'g $\alpha$ ) are stable, activation measurements are not possible. Thus the data base for comparison is limited.

Before these measurements were made, the only evaluation that was close to the data in the energy range above $20 \mathrm{MeV}$ was that of FZK/INPE [10]. After the present data were available to the evaluators, corrections were made in the reaction model calculation so that the ENDF/B-VII evaluation now follows the data well above $20 \mathrm{MeV}$. There still is a problem with that evaluation in the $7-13 \mathrm{MeV}$ range however.

\section{Summary}

The cross sections for producing hydrogen and helium have been measured for incident neutron energies from threshold to $100 \mathrm{MeV}$ on iron, chromium and tantalum by detecting the emitted charged particles: protons, deuterons, tritons, ${ }^{3} \mathrm{He}$ and alpha particles. For iron and chromium, the production cross sections for both hydrogen and helium rise from threshold

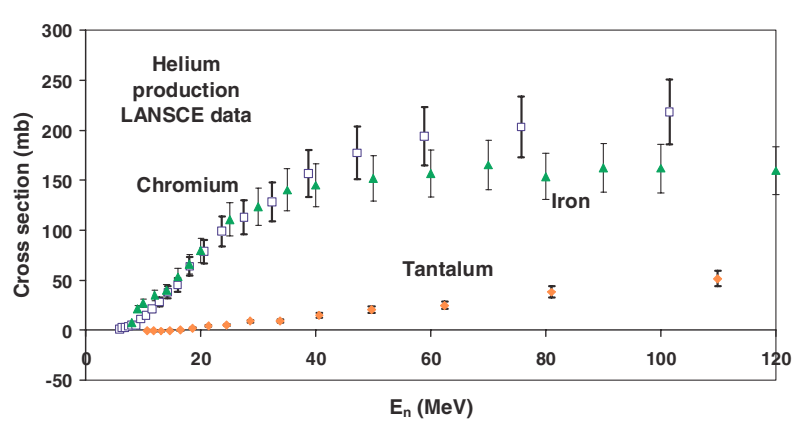

Fig. 3. Helium production by neutrons on iron, chromium, and tantalum for neutron energies up to $100 \mathrm{MeV}$.

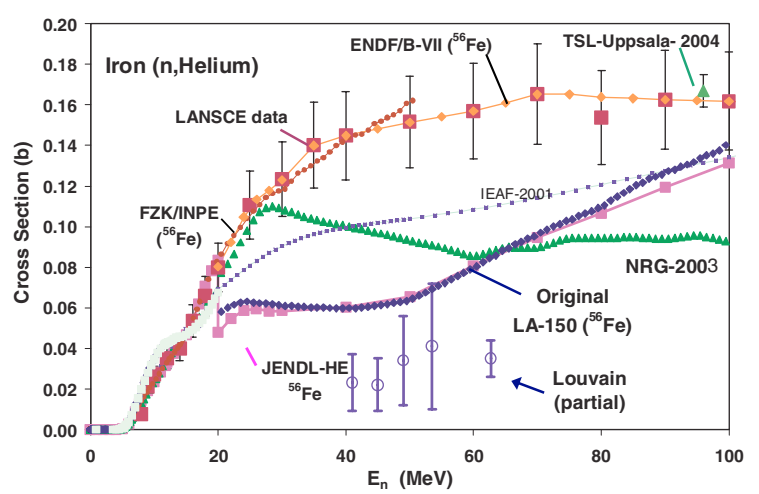

Fig. 4. Helium production cross sections for neutrons on iron from threshold to $100 \mathrm{MeV}$ compared with several evaluations. The $95 \mathrm{MeV}$ data point is from ref. [6]. The data from Louvain [7] do not include most of the alpha evaporation peak.

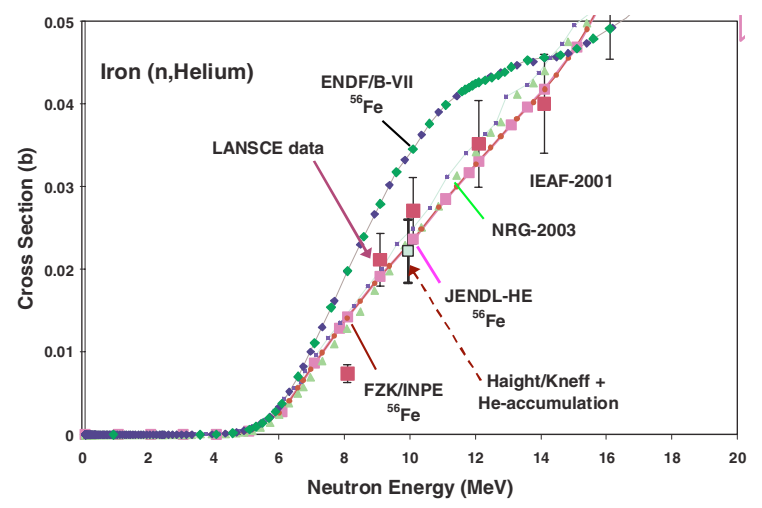

Fig. 5. Helium production by neutrons on iron below $16 \mathrm{MeV}$. The helium accumulation datum at $9.85 \mathrm{MeV}$ [7] is in agreement with the new data presented here and with some of the evaluated data libraries.

up to about $50 \mathrm{MeV}$, above which energy the cross sections are relatively flat. For tantalum the production cross sections for these gases also rise from threshold but continue to increase with increasing incident neutron energy to $100 \mathrm{MeV}$, the highest incident neutron energy investigated here. At all energies up to at least $100 \mathrm{MeV}$, the production cross sections on tantalum are significantly less than those on iron and chromium.

The author would like to thank Dr Terry Taddeucci for the MCNPX calculation of the neutron fluence. This work has benefited from the 
use of the Los Alamos Neutron Science Center at the Los Alamos National Laboratory. This facility is funded by the US Department of Energy and operated by Los Alamos National Security, LLC under contract DE-AC52 06NA25396.

\section{References}

1. P.W. Lisowski, C.D. Bowman, G.J. Russell, S.A. Wender, Nucl. Sci. Eng. 106, 208 (1990).

2. S.M. Grimes, C.E. Brient, F.C. Goeckner, F.B. Bateman, M.B. Chadwick, R.C. Haight, T.M. Lee, S.M. Sterbenz, P.G. Young, O.A. Wasson, H. Vonach, Nucl. Sci. Eng. 124, 271 (1996).

3. Goodfellow Corporation, Oakdale, PA 15071, USA.

4. S.A. Wender, S. Balestrini, A. Brown, R.C. Haight, C.M. Laymon, T.M. Lee, P.W. Lisowski, W. McCorkle,
R.O. Nelson, W. Parker, N.W. Hill, Nucl. Instrum. Meth. Phys. Res. A 336, 226 (1993).

5. T. Taddeucci (2007) (private communication).

6. F.R. Lecolley et al., International Conference on Nuclear Data for Science and Technology, Santa Fe, New Mexico, USA, Sept. 26-Oct. 1, 2004, edited by R.C. Haight, M.B. Chadwick, T. Kawano, P. Talou, AIP Conf. Proc. 769 (Melville, NY, 2005), p. 61 .

7. I. Slypen, N. Nica, A. Koning, E. Raeymackers, S. Benck, J.P. Meulders, V. Corcalciuc, J. Phys. G. Nucl. Part. Phys. 30, 45 (2004).

8. R.C. Haight, D.W. Kneff, B.M. Oliver, L.R. Greenwood, H. Vonach, Nucl. Sci. Eng. 124, 219 (1996).

9. S.M. Grimes, R.C. Haight, K.R. Alvar, H.H. Barschall, R.R. Borchers, Phys. Rev. C 19, 2127 (1979).

10. FZK/INPE: Forschungszentrum Karlsruhe - Obninsk Institute of Nuclear Power Engineering Evaluated Data Library. 